\title{
Local effective dynamics of quantum systems: A generalized approach to work and heat
}

\author{
Hendrik Weimer, Markus J. Henrich, Florian Rempp, Heiko Schröder, and Günter Mahler \\ Institute of Theoretical Physics I, University of Stuttgart, Pfaffenwaldring 57, 70550 Stuttgart, Germany*
}

(Dated: October 25, 2018)

\begin{abstract}
By computing the local energy expectation values with respect to some local measurement basis we show that for any quantum system there are two fundamentally different contributions: changes in energy that do not alter the local von Neumann entropy and changes that do. We identify the former as work and the latter as heat. Since our derivation makes no assumptions on the system Hamiltonian or its state, the result is valid even for states arbitrarily far from equilibrium. Examples are discussed ranging from the classical limit to purely quantum mechanical scenarios, i.e. where the Hamiltonian and the density operator do not commute.

PACS numbers: 05.30.-d, 03.65.-w, 05.70.Ln
\end{abstract}

The formulation of classical thermodynamics was one of the most important achievements of the $19^{\text {th }}$ century, as it allowed to investigate a large variety of phenomena, including the workings of thermodynamical machines. The first law of thermodynamics,

$$
\mathrm{d} U=\mathrm{d} W+\mathrm{d} Q
$$

combined with definitions for the infinitesimal change in work $\mathrm{d} W$ and heat $\mathrm{d} Q$ and the second law is all that is required for computing important quantities like the efficiency of a process.

In the quantum realm, the classification of work and heat is less clear. So far, it has mainly been based on the change of the total energy expectation value

$$
\mathrm{d} U=\mathrm{d} \operatorname{Tr}\{\hat{H} \hat{\rho}\}=\operatorname{Tr}\{\hat{\rho} \mathrm{d} \hat{H}+\hat{H} \mathrm{~d} \hat{\rho}\}
$$

and defining the first term as $\mathrm{d} W$ and the second as $\mathrm{d} Q$ [1-5]. However, such a classification can be problematic, for various reasons: For one, it is not obvious how to apply this definition to processes involving an internal transfer of work and heat, as is the case, e.g., in algorithmic cooling, a method to obtain highly polarized spins by applying a series of quantum gates [6]. Then, the microscopic foundation of (2) is rather unclear: As thermodynamic behavior may occur even in small quantum systems [7], it should, in principle, be possible to obtain $\mathrm{d} W$ and $\mathrm{d} Q$ even there. In the following, we will present an alternative definition that does not suffer from the problems above.

This letter is organized as follows. We first discuss the local effective dynamics of a bipartite quantum system. This dynamics of one part of the system is a reduced dynamics, which depend on the state of and the interaction with the rest of the system. Because we are interested in local properties of a part of the system, it is necessary to get a complete local description. In contrast to, e.g., a Markovian quantum master equation (see, e.g., [8]) the reduced dynamics cannot expected to be a set of closed differential equations. Based upon what an experimentalist might observe, we give a definition for the local energy. We then show that the change in this local energy can always be split into a part that correlates with a change in entropy and in a part which does not. Corresponding to classical thermodynamics, the former will be called "heat" and the latter "work". However, this definition for the local heat and work does not only depend on local properties, but on details of the whole system. We explicitly give formulas to calculate those local quantities once the time evolution of the full system is known. Finally, two examples will be given, for illustration.

While work and heat can thus always be introduced to characterize the energy exchange between two quantum subsystems, this splitting lacks, in general, the robustness and universality, which is typical for the corresponding thermodynamic concept. Additional investigations are needed to show under which conditions this work and heat may be identified with their thermodynamic counterparts. (This is reminiscent of entropy, which also can be defined for any quantum state, but its relationship to the thermodynamic variant requires further analysis.) However, it is necessary to develop generalized concepts for work and heat if one wants to analyze question requiring both quantum mechanics and thermodynamics, like when investigating the influence of coherence on the efficiency of a quantum machine.

We consider an autonomous bipartite system described by the Hamiltonian

$$
\hat{H}=\hat{H}_{A}+\hat{H}_{B}+\hat{H}_{A B},
$$

where $\hat{H}_{A}$ acts on subsystem $A$ and $\hat{H}_{B}$ on $B$, respectively. We now focus on the local properties of subsystem $A$ only, however our scheme would work also for subsystem $B$. The problem of local addressability has been discussed intensively in quantum computing [6]. A local measurement of work or heat will have to be be based on a coupling to a heat bath or work reservoir, respectively. (The energy exchanged with a bath must be heat, that with a "mechanical" reservoir work, by definition.) In 
TABLE I: Comparison of the scenarios when coupling a system to a heat bath or a work reservoir, depending on the interaction Hamiltonian.

\begin{tabular}{lll}
\hline \hline & work reservoir & heat bath \\
\hline$\hat{\sigma}_{x}$ & $\mathrm{~d} U=\mathrm{d} W=0$ & $\mathrm{~d} U=\mathrm{d} Q \neq 0$ \\
$\hat{\sigma}_{z}$ & $\mathrm{~d} U=\mathrm{d} W \neq 0$ & $\mathrm{~d} U=\mathrm{d} Q=0$ \\
\hline \hline
\end{tabular}

both cases the coupling will be realized by an interaction Hamiltonian $\hat{H}_{A M}$ of the type

$$
\hat{H}_{A M}=\sum_{i} \hat{A}_{i} \otimes \hat{M}_{i}
$$

where the operators $\hat{A}_{i}$ act only on subsystem $A$, and the $\hat{M}_{i}$ only on the measuring device $M$. Choosing the operators $\hat{A}_{i}$ may seem rather arbitrary, but can have important consequences on the observed values. For example, let us consider a system with an effective local Hamiltonian

$$
\hat{H}_{A}^{\mathrm{eff}}=\frac{\Delta E}{2} \hat{\sigma}_{z} .
$$

We now consider four cases: first, the system will be coupled to a work reservoir, which can always be modeled by a classical driver, as the work reservoir must always have zero entropy, by definition. We use $\hat{A}_{i}=\hat{A}=g \sin (\omega t) \hat{\sigma}_{x}$ and $\hat{A}=g \sin (\omega t) \hat{\sigma}_{z}$, respectively. For simplicity, we assume that the driving frequency is off-resonant with respect to the eigenfrequency of $\hat{H}_{A}^{\mathrm{eff}}$. Therefore, in the $\hat{\sigma}_{x}$ case there will be no transfer of energy, while in the $\hat{\sigma}_{z}$ case $\mathrm{d} U$ is non-zero, and all energy is transferred as work. We then repeat the process when coupling the system to a heat bath. Here, we have $\hat{A}=\lambda \hat{\sigma}_{x}$ and $\hat{A}=\lambda \hat{\sigma}_{z}$, respectively. In the $\hat{\sigma}_{x}$ case, this results in system relaxing to a canonical state $\hat{\rho}_{A}=Z^{-1} \exp \left(-\beta \hat{H}_{A}^{\text {eff }}\right)$, with $\beta$ being the inverse temperature. However, the $\hat{\sigma}_{z}$ coupling results in a pure dephasing of the system, i.e, $\mathrm{d} U=\mathrm{d} Q=0$. A summary of the possible combinations is shown in Table I.

The concrete physical realization thus defines a local effective measurement basis (LEMBAS), which provides a reference for all measurements of work or heat. Which choice of basis is the "correct" one, cannot be decided by the LEMBAS principle. However, there are typically only a few choices which are physically reasonable, which then can be further checked for consistency. A constructive procedure that would allow to identify the correct basis is beyond the scope of this letter.

In the following we do not consider the effect of the actual measurement on the dynamics of the system. We only assume that some fixed basis has been chosen as required by the LEMBAS principle, and then compute work and heat with respect to this basis. Note that this procedure is similar to a hypothetic von Neumann measurement - the probability for each outcome can be cal- culated without including the measurement device in the dynamics.

We now define the infinitesimal work $\mathrm{d} W_{A}$ performed on $A$ as the change in its internal energy $\mathrm{d} U_{A}$ that does not change its local von Neumann entropy, i.e.

$$
\mathrm{d} S_{A}=0 \Leftrightarrow \mathrm{d} W_{A}=\mathrm{d} U_{A} .
$$

The remainder is defined as the infinitesimal heat $\mathrm{d} Q$. It is important to note that work and heat will then turn out to be basis-dependent quantities as they depend on the choice of the measurement basis.

The dynamics of the subsystem $A$ is given by the Liouville-von Neumann equation

$$
\frac{\partial}{\partial t} \hat{\rho}_{A}=-\mathrm{i}\left[\hat{H}_{A}+\hat{H}_{A}^{\mathrm{eff}}, \hat{\rho}_{A}\right]+\mathcal{L}_{\text {inc }}(\hat{\rho}),
$$

where $\hat{\rho}_{A}$ is the reduced density operator of $A, \hat{H}_{A}^{\mathrm{eff}}$ is an effective Hamiltonian (see below) describing the unitary dynamics induced by $B$ on $A$, and $\mathcal{L}_{\text {inc }}$ is a superoperator describing incoherent processes, which may derive from the environment of the total bipartite system (if present), but here, in particular, from the influence of $B$ on $A$. Since $\mathcal{L}_{\text {inc }}$ is, in general, a function of the density operator of the full system, Eq. (7) is typically not a closed differential equation for $\hat{\rho}_{A}$.

In the following we choose the energy basis of subsystem $A$ as the measurement basis, so that only the parts of the total effective Hamiltonian $\hat{H}_{A}^{\text {eff }}$ that commute with $\hat{H}_{A}$ contribute to the described type of experiment. To find this part $\hat{H}_{1}^{\text {eff }}$, we expand $\hat{H}_{A}^{\text {eff }}$ in the transition operator basis defined by the energy eigenstates $\{|j\rangle\}$ of $\hat{H}_{A}$ :

$$
\hat{H}_{A}^{\mathrm{eff}}=\sum_{j k}\left(\hat{H}_{A}^{\mathrm{eff}}\right)_{j k}|j\rangle\langle k|
$$

For this operator basis, we have

$$
\left[|j\rangle\langle k|, \hat{H}_{A}\right]=\omega_{k j}|j\rangle\langle k|,
$$

where $\omega_{k j}$ is the difference between the energy eigenvalues of the states $|k\rangle$ and $|j\rangle$, and therefore $\omega_{j j}=0$ for non-degenerate energy eigenvalues. Now, we define

$$
\hat{H}_{1}^{\mathrm{eff}}=\sum_{j}\left(\hat{H}_{A}^{\mathrm{eff}}\right)_{j j}|j\rangle\langle j|
$$

which is the diagonal part of $\hat{H}_{A}^{\text {eff }}$. From Eq. (9), we see that no non-zero linear combination of transition operators with $j \neq k$ can ever commute with $\hat{H}_{A}$ and therefore, neither $\hat{H}_{2}^{\text {eff }}=\hat{H}_{A}^{\text {eff }}-\hat{H}_{1}^{\text {eff }}$, nor any part of it commutes with $\hat{H}_{A}$. Hence, $\hat{H}_{1}^{\text {eff }}$ is the part contributing to the measurement, as required, and we have

$$
\left[\hat{H}_{1}^{\text {eff }}, \hat{H}_{A}\right]=0,\left[\hat{H}_{2}^{\text {eff }}, \hat{H}_{A}\right] \neq 0 .
$$

The latter inequality holds except for the case where $\hat{H}_{2}^{\text {eff }}=0$. An analogous result can be achieved in the 
case of degenerate eigenvalues of $\hat{H}_{A}$ but has been omitted for the sake of brevity and clarity.

If a measurement of the local energy is performed in the energy eigenbasis of $\hat{H}_{A}$ the corresponding operator is

$$
\hat{H}_{A}^{\prime}=\hat{H}_{A}+\hat{H}_{1}^{\mathrm{eff}} .
$$

Therefore the change in internal energy within $A$ is given by

$$
\mathrm{d} U_{A}=\frac{\mathrm{d}}{\mathrm{d} t} \operatorname{Tr}\left\{\hat{H}_{A}^{\prime} \hat{\rho}_{A}\right\} \mathrm{d} t=\operatorname{Tr}\left\{\dot{\hat{H}}^{\prime} \hat{\rho}_{A}+\hat{H}_{A}^{\prime} \dot{\rho}_{A}\right\} \mathrm{d} t .
$$

Using (7) and assuming $\hat{H}_{A}$ to be time-independent leads to

$$
\mathrm{d} U_{A}=\operatorname{Tr}\left\{\dot{\hat{H}_{1}^{\mathrm{eff}}} \hat{\rho}_{A}-\mathrm{i}\left[\hat{H}^{\prime}, \hat{H}_{2}^{\mathrm{e} f f}\right] \hat{\rho}_{A}+\hat{H}_{A}^{\prime} \mathcal{L}_{i n c}(\hat{\rho})\right\} \mathrm{d} t,
$$

where the cyclicity of the trace has been used. Observing that the dynamics induced by the first two terms is unitary, we arrive at

$$
\begin{aligned}
\mathrm{d} W_{A} & =\operatorname{Tr}\left\{\dot{\hat{H}}_{1}^{\text {eff }} \hat{\rho}_{A}-\mathrm{i}\left[\hat{H}^{\prime}, \hat{H}_{2}^{\text {eff }}\right] \hat{\rho}_{A}\right\} \mathrm{d} t \\
\mathrm{~d} Q_{A} & =\operatorname{Tr}\left\{\hat{H}_{A}^{\prime} \mathcal{L}_{i n c}(\hat{\rho})\right\} \mathrm{d} t .
\end{aligned}
$$

In this sense, it is possible to define heat and work for any quantum mechanical process, regardless of the type of dynamics or the states involved.

In order to actually compute $\mathrm{d} W$ and $\mathrm{d} Q$ the effective Hamiltonian $\hat{H}_{A}^{\text {eff }}$ is required. By starting with the Liouville-von Neumann equation for the full system

$$
\frac{\partial}{\partial t} \hat{\rho}=-\mathrm{i}[\hat{H}, \hat{\rho}]
$$

and taking the partial trace over $B$ (see e.g. [8]) yields

$$
\frac{\partial}{\partial t} \hat{\rho}_{A}=\operatorname{Tr}_{B}\left\{\left[\hat{H}_{A}+\hat{H}_{B}+\hat{H}_{A B}, \hat{\rho}\right]\right\} .
$$

Using some theorems on partial traces shows that terms involving $\hat{H}_{B}$ vanish and $\hat{H}_{A}$ generates the local dynamics in $A$. For dealing with the terms involving $\hat{H}_{A B}$ we first split the density operator as

$$
\hat{\rho}=\hat{\rho}_{A} \otimes \hat{\rho}_{B}+\hat{C}_{A B},
$$

where $\hat{\rho}_{A, B}$ are the reduced density operators for $A$ and $B$, respectively, and $\hat{C}_{A B}$ is the operator describing the correlations between both subsystems. Since the factorization approximation is exact for the first term, we can write (cf. [9])

$$
\operatorname{Tr}_{B}\left\{\left[\hat{H}_{A B}, \hat{\rho}_{A} \otimes \hat{\rho}_{B}\right]\right\}=\left[\hat{H}_{A}^{\mathrm{eff}}, \hat{\rho}_{A}\right],
$$

where $\hat{H}_{A}^{\text {eff }}$ is given by

$$
\hat{H}_{A}^{\mathrm{eff}}=\operatorname{Tr}_{B}\left\{\hat{H}_{A B}\left(\hat{1}_{A} \otimes \hat{\rho}_{B}\right)\right\} .
$$

Now we show that the processes generated by $\left[\hat{H}_{A B}, \hat{C}_{A B}\right]$ cannot result in unitary dynamics, but will always change the local von Neumann entropy $S_{A}$. In order to prove this, we convince ourselves that its time derivative is non-zero, i.e,

$$
\dot{S}_{A}=-\operatorname{Tr}\left\{\left[\hat{H}_{A B}, \hat{C}_{A B}\right] \log \hat{\rho}_{A} \otimes \hat{1}_{B}\right\} \neq 0 .
$$

Therefore, any dynamics generated by this term results in a contribution to $\mathcal{L}_{\text {inc }}$. If the dynamics of the full system was unitary, we would thus simply have

$$
\mathcal{L}_{i n c}=-i \operatorname{Tr}_{B}\left\{\left[\hat{H}_{A B}, \hat{C}_{A B}\right]\right\} .
$$

as the total incoherent term.

How are these alternative definitions of heat and work linked to Eq. (2)? It is easy to check that for $\hat{H}_{2}^{\text {eff }}=$ 0 the definitions are compatible. A pertinent example refers to a quasistatic process within the system $A-B$ in thermal equilibrium at temperature $T$. From the Gibbs fundamental relation it is known that

$$
\mathrm{d} S_{A}=\frac{1}{T_{A}} \mathrm{~d} Q_{A} .
$$

Using now the definition given for the heat in (16) we get

$$
\mathrm{d} S_{A}=\frac{1}{T_{A}^{*}} \operatorname{Tr}\left\{\hat{H}_{A}^{\prime} \mathcal{L}_{\mathrm{inc}}(\hat{\rho})\right\} \mathrm{d} t .
$$

Here, $T_{A}^{*}$ specifies a parameter to be associated with the local temperature. This parameter follows from the derivation of the entropy $S_{A}$ from (22) combined with (25)

$$
\begin{aligned}
-\operatorname{Tr}\left\{\mathcal{L}_{\text {inc }}(\hat{\rho}) \log \hat{\rho}_{A}\right\} & =\frac{1}{T_{A}^{*}} \operatorname{Tr}\left\{\hat{H}_{A}^{\prime} \mathcal{L}_{\text {inc }}(\hat{\rho})\right\} \\
T_{A}^{*} & =\frac{\operatorname{Tr}\left\{\hat{H}_{A}^{\prime} \mathcal{L}_{\text {inc }}(\hat{\rho})\right\}}{\operatorname{Tr}\left\{\mathcal{L}_{\text {inc }}(\hat{\rho}) \log \hat{\rho}_{A}\right\}} .
\end{aligned}
$$

For canonical states $\hat{H}_{A}^{\prime}$ commutes with $\hat{\rho}_{A}$ and $\mathcal{L}_{\text {inc }}\left(\hat{\rho}_{A}\right)$, and by using Eq. (14) it can be seen that Eq. (26) is equivalent to the classical definition

$$
T_{A}^{*}=T_{A}=\frac{\partial U_{A}}{\partial S_{A}} .
$$

However, $T_{A}^{*}$ is not necessarily equal to the global temperature $T$ of the full system due to the interaction between the individual subsystems inducing correlations $[10,11]$.

Using the LEMBAS principle, it is now possible to investigate work and heat also in non-standard physical scenarios. First we consider a two-level atom with a local Hamiltonian $\hat{H}_{A}$ interacting with a laser field (subsystem $B)$. In the semiclassical treatment of the radiation field emitted by a laser the respective Hamiltonian is given by

$$
\hat{H}=\hat{H}_{A}+\hat{H}_{A}^{\mathrm{eff}}=\frac{\Delta E}{2} \hat{\sigma}_{z}+g \sin (\omega t) \hat{\sigma}_{x},
$$




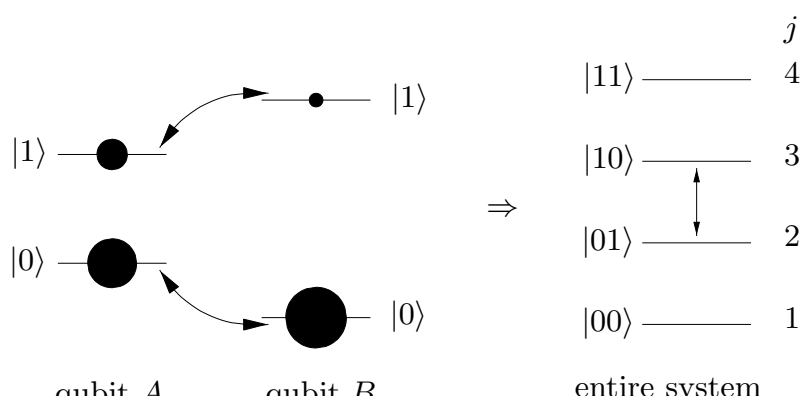

qubit $A \quad$ qubit $B$

FIG. 1: Scheme of the SWAP gate and the accounting $\pi$-pulse.

where $g$ is the coupling strength and $\omega$ the laser frequency. In the rotating wave approximation the Hamiltonian can be made time-independent. We investigate the situation where the atom is initially in a thermal state described by the density operator

$$
\hat{\rho}_{A}(0)=Z^{-1} \exp \left(-\beta \hat{H}_{A}\right),
$$

with $Z$ being the partition function and $\beta$ the inverse temperature. The time-evolution of the density operator $\hat{\rho}_{A}(t)$ can be obtained by switching to the rotating frame and diagonalizing the Hamiltonian.

Since (28) is already an effective description for $A$, we can directly compute đ $W_{A}$ and đ $Q_{A}$ once we know $\hat{\rho}_{A}(t)$, resulting in

$$
\begin{aligned}
\mathrm{d} W_{A}(\delta, g) & =\frac{\Delta E g^{2}}{2 \Omega} \tanh \frac{\beta \Delta E}{2} \sin \Omega t \\
\mathrm{~d} Q_{A} & =0,
\end{aligned}
$$

where $\Omega=\sqrt{g^{2}+\delta^{2}}$ is the Rabi frequency and $\delta=$ $\omega-\Delta E / \hbar$ is the detuning from the resonance frequency. $\mathrm{d} W_{A}$ is the energy stored in $A$ that could be retrieved after the preparing field has been switched off at time $\Omega t=\pi / 2$. For comparison, using the previously used definition for the work [Eq. (2)] one was led to

$$
\mathrm{d} W_{A}(\delta, g)=\frac{(\Delta E+\delta) g^{2}}{2 \Omega} \tanh \frac{\beta \Delta E}{2} \sin \Omega t .
$$

Comparison of the two results shows that in the latter case the maximum is not at the resonance frequency $(\delta=$ $0)$, i.e., only our generalized approach is able to produce the correct physical result.

As a second example we investigate the implications of our generalized concept in the context of algorithmic cooling. Recently an algorithm published by Boykin et al. [12] has found experimental realization by Baugh et al. [13] and its thermodynamic properties where investigated by Rempp et al. [14]. Of course, not all qubits [i. e. two level systems (TLS) used for quantum computing] are cooled down this way, some, the so called auxiliary qubits, are heated up as well and thus have to be discarded or coupled to the environment to work again as auxiliaries. The simplest system to perform algorithmic cooling consists of two noninteracting TLS with different Zeeman splitting $\left(\Delta E_{A}<\Delta E_{B}\right)$ at the same initial inverse temperature $\beta_{i}$. The cooling algorithm is a single SWAP gate, which interchanges the occupation probabilities between the two spins (see Fig. 1); this implies a final inverse temperature $\beta_{f}=\left(\Delta E_{B} / \Delta E_{A}\right) \beta_{i}$ of the cooled spin. This one-step cooling of a finite system (here one spin) reminds of a refrigerator and thus should be characterized by properties like the efficiency $\eta=Q / W$. To distinguish between work $W$ and heat $Q$ one needs to apply the local treatment described above. If one would just use Eq. (2) the heat would be $Q=0$ because the entropy of the total system does not change. Therefore, it is necessary to apply our generalized approach.

Again, in the semi-classical treatment of the subsystem $C$ inducing the SWAP gate between the $|01\rangle$ and $|10\rangle$ levels the Hamiltonian in the rotating wave approximation reads (index $j$ shown in Fig. 1)

$$
\begin{aligned}
\hat{H}(t) & =\hat{H}_{A B}+\hat{H}_{\mathrm{int}}(t)=\sum_{j=1}^{4} E_{j} \hat{P}_{j j} \\
& +\frac{1}{2} g\left(\mathrm{e}^{\mathrm{i}\left(E_{3}-E_{2}\right) t} \hat{P}_{23}+\mathrm{e}^{-\mathrm{i}\left(E_{3}-E_{2}\right) t} \hat{P}_{32}\right)
\end{aligned}
$$

with $\sum_{j=1}^{4} E_{j} \hat{P}_{j j}=\sum_{\mu=A, B} \Delta E_{\mu} \hat{\sigma}_{\mu}^{z}$ representing the spectrum of the two uncoupled spins. To solve the equations of motion of this Hamiltonian we transform into the rotating basis to get rid of the time-dependence of the Hamiltonian [15]

$$
\hat{H}_{\text {rot }}=\hat{U}_{\text {rot }}(t) \hat{H}(t) \hat{U}_{\text {rot }}^{-1}(t) .
$$

Thus we are able to calculate the infinitesimal change of work $\mathrm{d} W_{A B}$ done by the subsystem $C$ on the two-spin system by means of Eq. (15),

$$
\mathrm{d} W_{A B}=\frac{g}{2} Z^{-1}\left(\mathrm{e}^{\beta_{i} \Delta E_{A}}-\mathrm{e}^{\beta_{i} \Delta E_{B}}\right)\left(\Delta E_{A}-\Delta E_{B}\right) \sin g t
$$

where $Z$ is the partition function of the system. To get the work $W_{A B}$ induced by a SWAP we have to integrate over a half period of the Rabi oscillation. Because there is no detuning this is the same result as if calculated directly via Eq. (2). As in the previous example đ $Q_{A B}=$ 0 , i.e., subsystem $C$ only imparts work. Interestingly enough, the situation changes when we only look at $A$ : To compute the transferred heat $Q_{A}$ we have to apply the LEMBAS principle to investigate the local change of energy that is related to a change of the local entropy. We use (21) to find the effective Hamiltonian

$$
\begin{aligned}
\hat{H}_{A}^{\mathrm{eff}}= & -\hat{\sigma}^{0}\left\{\left[\mathrm{e}^{\beta_{i}\left(\Delta E_{A}+\Delta E_{B}\right)}-1\right] Z^{-1} \Delta E_{B}\right. \\
& \left.+\left(\mathrm{e}^{\beta_{i} \Delta E_{B}}-\mathrm{e}^{\beta_{i} \Delta E_{A}}\right) Z^{-1} \Delta E_{B} \cos (g t)\right\} \\
& +\hat{\sigma}^{z} \Delta E_{A} .
\end{aligned}
$$


From the fact that $\left[\hat{\sigma}^{0}, \hat{\sigma}^{z}\right]=0$ it follows that $\hat{H}_{A}^{\text {eff }}=\hat{H}_{A}^{\prime}$ and

$$
\frac{\mathrm{d}}{\mathrm{d} t} \hat{\rho}_{A}(t)=\frac{\mathrm{d}}{\mathrm{d} t} \operatorname{Tr}_{B}\{\hat{\rho}(t)\}=\mathcal{L}_{\text {inc }} .
$$

Using Eq. (16) yields

$$
\mathrm{d} Q_{A}=-\frac{g}{2} Z^{-1}\left(\mathrm{e}^{\beta_{i} \Delta E_{A}}-\mathrm{e}^{\beta_{i} \Delta E_{B}}\right) \Delta E_{A} \sin (g t),
$$

which, again, has to be integrated over a half period of the Rabi oscillation to get $Q_{A}$. This is the heat that would have to flow back from the original heat bath to let subsystem $A$ return to its initial state. The SWAP gate thus has the engine efficiency $\eta_{A}$

$$
\eta_{A}=-\frac{Q_{A}}{W_{A B}}=\frac{\Delta E_{A}}{\Delta E_{A}-\Delta E_{B}} .
$$

This is the same result as found for the quantum Otto process in the heat pump mode [4].

In summary we have shown the that exchange of energy between two quantum systems can always be split into work and heat, based on local effective dynamics. We have demonstrated how to obtain this local effective dynamics, once the dynamics of the full system is known. As this involves the choice of a measurement basis, those quantities are basis-dependent. The choice of basis remains a subtle problem; a detailed analysis of the environment should eventually give a unique answer, just as in case of a measuring apparatus with respect to its actual measurement basis. However, the examples presented show that our generalized approach can lead to concrete and sensible results, which is not always the case for the previously used one. The LEMBAS principle should help to investigate thermodynamic aspects at the borderline between full-fledged quantum dynamics and thermodynamics proper.
We thank M. Michel, A. Kettler, G. Reuther, H. Schmidt, and J. Teifel for fruitful discussions. Financial support by the Deutsche Forschungsgemeinschaft is gratefully acknowledged.

* Electronic address: hweimer@itp1.uni-stuttgart.de

[1] R. Alicki, J. Phys. A 12, L103 (1979).

[2] R. Kosloff and M. A. Ratner, J. of Chem. Phys. 80, 2352 (1984).

[3] T. D. Kieu, Phys. Rev. Lett. 93, 140403 (2004).

[4] M. J. Henrich, M. Michel, and G. Mahler, Europhys. Lett. 76, 1057 (2006).

[5] M. J. Henrich, M. Michel, and G. Mahler, Phys. Rev. E 75, 051118 (2007).

[6] M. A. Nielsen and I. L. Chuang, Quantum computation and quantum information (Cambridge University Press, Cambridge, 2000).

[7] J. Gemmer, M. Michel, and G. Mahler, Quantum Thermodynamics, Lecture Notes in Physics, Vol. 657 (Springer, Berlin, 2004).

[8] H.-P. Breuer and F. Petruccione, The Theory of Open Quantum Systems (Oxford University Press, Oxford, 2002).

[9] J. Gemmer and G. Mahler, Eur. Phys. J. D 17, 385 (2001).

[10] M. Hartmann, G. Mahler, and O. Hess, Phys. Rev. Lett. 93, 080402 (2004).

[11] M. Hartmann, G. Mahler, and O. Hess, Phys. Rev. E 70, 066148 (2004).

[12] P. Boykin, T. Mor, V. Roychowdhury, F. Vatan, and R. Vrijen, Proc. Natl. Acad. Sci. USA, 99, 3388 (2002).

[13] J. Baugh, O. Moussa, C. Pyan, A. Nayak, and R. Laflamme, Nature (London) 438, 470 (2005).

[14] F. Rempp, M. Michel, and G. Mahler, Phys Rev. A 76, 032325 (2007).

[15] G. Mahler and V. A. Weberruß, Quantum Networks (Springer, Berlin, 1998). 